\title{
Accuracy and repeatability of wrist joint angles in boxing using an electromagnetic tracking system
}

\author{
Ian T. Gatt ${ }^{1,3}$ (1) $\cdot$ Tom Allen $^{2} \cdot$ Jon Wheat ${ }^{3}$
}

Published online: 7 December 2019

(c) The Author(s) 2019

\begin{abstract}
The hand-wrist region is reported as the most common injury site in boxing. Boxers are at risk due to the amount of wrist motions when impacting training equipment or their opponents, yet we know relatively little about these motions. This paper describes a new method for quantifying wrist motion in boxing using an electromagnetic tracking system. Surrogate testing procedure utilising a polyamide hand and forearm shape, and in vivo testing procedure utilising 29 elite boxers, were used to assess the accuracy and repeatability of the system. 2D kinematic analysis was used to calculate wrist angles using photogrammetry, whilst the data from the electromagnetic tracking system was processed with visual 3D software. The electromagnetic tracking system agreed with the video-based system (paired $t$ tests) in both the surrogate $\left(<0.2^{\circ}\right)$ and quasistatic testing $\left(<6^{\circ}\right)$. Both systems showed a good intraclass coefficient of reliability (ICCs $>0.9$ ). In the punch testing, for both repeated jab and hook shots, the electromagnetic tracking system showed good reliability (ICCs $>0.8$ ) and substantial reliability (ICCs $>0.6$ ) for flexion-extension and radial-ulnar deviation angles, respectively. The results indicate that wrist kinematics during punching activities can be measured using an electromagnetic tracking system.
\end{abstract}

Keywords Electromagnetic tracking system $\cdot$ Boxing $\cdot$ Sports $\cdot$ Wrist $\cdot$ Kinematics

\section{Introduction}

Injuries to the hand and wrist are common in boxing, accounting for $16-35 \%$ of all injuries in training and competition $[1,2]$, with the highest rate observed in competition (347 injuries per $1000 \mathrm{~h}$ ) versus training $(<0.5$ injuries per $1000 \mathrm{~h}$ ) [1]. Further, total days lost to training and overall duration are greater for the hand and wrist than any other body part [2]. The wrist is the most distal joint of the upper limb, allowing movements necessary for functional activities

This article is a part of Topical Collection in Sports Engineering on Measuring Behavior in Sport and Exercise, edited by Dr. Tom Allen, Dr. Robyn Grant, Dr. Stefan Mohr and Dr. Jonathan Shepherd.

Ian T. Gatt

ian.gatt@eis2win.co.uk

1 Physiotherapy Department, English Institute of Sport, Sheffield, UK

2 Sports Engineering Research Team, Manchester Metropolitan University, Manchester, UK

3 Centre for Sports Engineering Research, Sheffield Hallam University, Sheffield, UK of daily living. These movements include flexion, extension, ulnar deviation, and radial deviation [3]. Knowledge of wrist kinematics during the impact phase of punching is required to understand injuries at the wrist in boxing.

Studies investigating the kinematics of boxing have provided information on the range of motion occurring at the shoulder and elbow joints, but not the wrist $[4,5]$. These studies used reflective surface markers, placed directly on the skin, and camera-based motion capture system with the results interpreted using Cardan angles, an approach that has been widely described in the literature [6-10]. In a similar manner, reflective surface markers have also been used to measure wrist kinematics during the activities of daily living [11-13]. In boxing, however, placing markers on the skin is not feasible, as bandages and gloves cover the hand and wrist. Placing markers on the glove would not be practicable, as its movement can differ from that of the underlying wrist joint.

Other equipment to measure wrist motion includes electro-goniometers or electromagnetic tracking systems. An advantage of both systems over visual tracking methods is that the receivers do not require direct line-of-sight to sensors placed on the skin, which is important considering 
boxers wear gloves. Electro-goniometers have been used to measure wrist motions during activities of daily living $[14,15]$, and in snowboarding to assess wrist extension during falls, which is another sport where wrist injuries are common [16] and gloves are worn [17]. Electromagnetic receivers have also been described in the assessment of wrist motions, in addition to other joints of the human body [11, 18-21]. Electro-goniometers are prone to measurement errors particularly due to crosstalk [22, 23], so equipment selection is important [24], as is precise alignment with the wrist joint anatomy. Further, electro-goniometers can incur damage from high and repetitive forces, an important consideration with boxing [25].

Receivers used in electromagnetic systems do not require precise alignment as those used in electro-goniometers [18, 19], although careful selection and identification of reference points on anatomical landmarks is required. Electromagnetic systems are also affected by ferromagnetic materials which can disturb the local magnetic field and, therefore, the position and orientation estimation [26], but a simple solution is to avoid using the systems near large metal objects. Therefore, electromagnetic tracking systems are potentially viable for assessing wrist kinematics during punching, yet the accuracy and repeatability have not been reported.

Electromagnetic tracking systems have certain limitations which should be considered; (a) specialist expertise is required for both use and analysis, and (b) data is not live and requires time for analysis. Conversely, inertial (accelerometers or gyroscopes) sensors can be easy to use and provide real time feedback. Inertial sensors are an emerging technology, which have been used in boxing to measure shot velocities and quantify fatigue during training [27-29]. Human motion analysis studies are evolving from the use of single sensor units (i.e. accelerometers or gyroscopes) towards using more complex unit sensing devices, such as inertial and magnetic measurements units known as wearable inertia measurable units (IMUs), to compensate for angle measurement errors [30, 31]. Angle measurement errors observed during data collection comprise mainly technical problems, such as transmission lag [32]. Additionally, the signals can be contaminated by noise introduced in the acceleration or magnetic signals, nature of the sensors, or human motion artefacts derived from sensor placement [30, 32]. Wearable sensors also need to handle joints involving more than one degree-of-freedom as multi-plane movements not only require the capability of tracking motion in different axes, but also the ability of removing bias that the movement in a different axis might have on a tracked axis $[6,31]$. Compared to single sensor units more complex unit sensing devices such as IMUs, which are being used to compensate for angle measurement errors and multiplane movements, require specialist expertise from diverse fields of knowledge making the use of these devices more complex [30].
Compared to other systems, electromagnetic tracking system was deemed the most applicable for investigating wrist kinematics during punching in boxing. The aim of this study was to investigate the accuracy and repeatability of an electromagnetic tracking system in measuring wrist motion during punching in boxing. This study included three components; (a) a mechanical surrogate-based investigation, using a polyamide hand and forearm shape surrogate, (b) an in vivo quasi-static measurement of the wrist, and (c) an in vivo measurement of the wrist during boxing punching activities.

\section{Method}

An electromagnetic tracking system (Polhemus, Colchester, VT, USA), with 6-degree-of-freedom (DoF) position and orientation receivers was used to record kinematic data at a sampling rate of $240 \mathrm{~Hz}$ using a custom-written software. Two and three receivers were, respectively, fixed to the surrogate and participants of this study. Segment coordinate systems were defined, using a digital stylus, based on eight non-anatomical landmarks for the surrogate (Fig. 1); four for the moving component (hand) and four for the non-mobile component (forearm), and 11 anatomical landmarks for the participants (Fig. 2). The anatomical landmarks were Hand; Head of 2nd Metacarpal Bone, Base of 2nd Metacarpal Bone, Head of 5th Metacarpal Bone, Base of 5th Metacarpal bone, Forearm; Styloid Process of Radius, $7 \mathrm{~cm}$ proximal to Styloid process of Radius, Head of Ulna, $7 \mathrm{~cm}$ proximal to Head of Ulna, and Arm; Medial Epicondyle of Humerus, Lateral Epicondyle of Humerus, Mid-Acromion of Scapula. Segment coordinate systems were embedded in the left upper limb segments, defined based on the location of the anatomical markers such that the $x$-, $y$ - and $z$-axis were medio-lateral, anterio-posterior and longitudinal, respectively. The orientation of the hand relative to the wrist was defined using Cardan angles (xyz rotation sequence), to

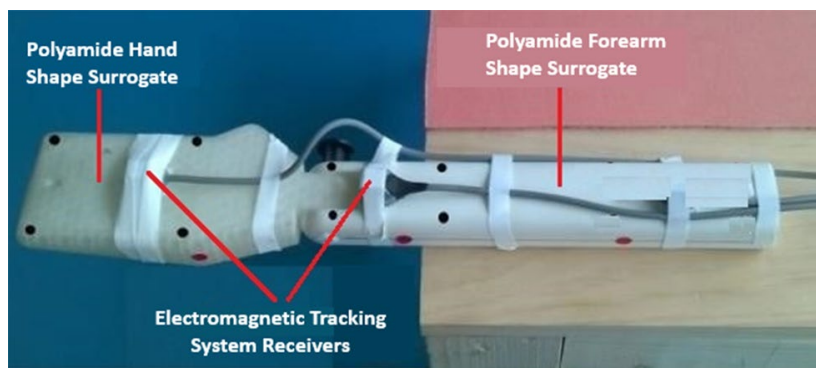

Fig. 1 Electromagnetic tracking system receivers $($ grey $\times 2)$, virtual markers (black $\times 8$ ), and self-adhesive markers (red $\times 3$ ) placement for the surrogate (colour figure online) 


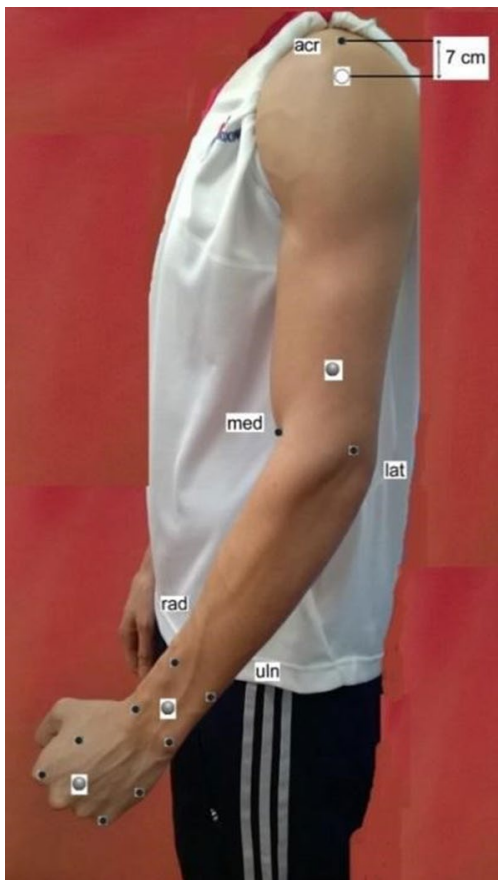

Fig. 2 Electromagnetic tracking system receivers (grey $\times 3$ ) and virtual markers (black $\times 11$ ) placement for the upper limb. Joint centre is dotted (shoulder joint). The virtual markers were digitised more medial and lateral for the actual study than observed on this figure. The current placement is solely provided as visual reference of the markers

determine wrist flexion-extension and radial-ulnar deviation angles $[6,8-10,12,33]$.

\subsection{Surrogate testing}

Two electromagnetic tracking system receivers were attached to a polyamide hand and forearm shape surrogate allowing for one DoF (flexion and extension). The surrogate was constructed with additive manufacturing using dimension guidelines from EN 14120; 2003 on wrist protectors [34]. One receiver was attached to the hand (mobile section), whilst another receiver was attached at the forearm (fixed section) using double-sided adhesive tape and zinc oxide tape (W $1.25 \mathrm{~cm})$.

Multiple positions of wrist angle were determined during three testing sessions; one to assess accuracy and another two for test-retest reliability. The surrogate hand was initially placed at an angle of $-90^{\circ}$ of extension and moved to a predetermined angle, where it was then held for approximately $5 \mathrm{~s}$ using wooden blocks ( $\mathrm{L} 10 \mathrm{~cm} \times \mathrm{W} 9 \mathrm{~cm} \times \mathrm{H}$ $4 \mathrm{~cm}$ ) positioned over a wooden rig ( $45 \mathrm{~cm} \times \mathrm{W} 14 \mathrm{~cm} \times \mathrm{H}$ $84 \mathrm{~cm})$. The hand was then returned to the initial position of $-90^{\circ}$. This procedure was performed for six wrist angle positions; three in extension $\left(-27^{\circ},-42.5^{\circ},-51^{\circ}\right)$ and three in flexion $\left(14.5^{\circ}, 28^{\circ}, 42^{\circ}\right)$. It is suggested that repeatability or reliability of an instrument and procedures is determined when the measurement tests are separated by short time intervals, also defined as a test-retest study design [35]. To measure test-retest reliability the system was recalibrated, the same six wrist angle positions performed, and data collected again. This re-test procedure was repeated one more time. Wrist angles were recorded at $60 \mathrm{fps}$ using a digital camcorder (Panasonic HC-V550), positioned with the image plane parallel to the plane of motion of the surrogate hand, from a distance of $150 \mathrm{~cm}$. Three self-adhesive coloured markers ( $6 \mathrm{~mm}$ diameter) positioned on the side of the surrogate were digitised in the video footage (Kinovea, open licence 0.8 .15 ) to calculate wrist angle.

\subsection{In-vivo testing}

\subsubsection{Participants}

To be included in the study, boxers were at an elite level, with no upper extremity symptoms. Selected participants were 29 Great Britain Boxers forming part of the National Olympic Squad (23 men and 6 women). Characteristics (mean \pm standard deviation) were as follows: age $24 \pm 4$ years (range 19-34 years), stature $178 \pm 10 \mathrm{~cm}$ (range $160-198 \mathrm{~cm}$ ), and mass $71 \pm 17 \mathrm{~kg}$ (range $50-114 \mathrm{~kg}$ ). All participants were right-arm dominant and orthodox stance boxers (left hand leading). The movements and experimental protocol were explained verbally. All participants received written information about the study and provided informed consent before testing. The study protocols were approved by Sheffield Hallam University Research Ethics Committee (Ref No HWB-S\&E-42).

\subsubsection{Quasi-static testing}

Three electromagnetic tracking system receivers were secured to the left upper arm, forearm and hand of the participants using double-sided adhesive tape, zinc oxide tape (W $1.25 \mathrm{~cm}$ ), and elastic bandaging ( $2.5 \mathrm{~cm}$ cohesive). At the hand and forearm, closed cell latex foam squares (Hapla Swanfoam, Cuxson Gerrard \& Co. Ltd.) were used as padding between the plastic receivers and bone prominence (L $2.5 \mathrm{~cm} \times \mathrm{W} 2.5 \mathrm{~cm} \times \mathrm{H} 0.3 \mathrm{~cm}$ ). The transmitter (source box) was elevated $1 \mathrm{~m}$ off the ground. In accordance with the manufacturer's guidelines, this provided detection of the magnetic signal generated by the transmitter within the hemispherical range radius required for all testing $(150 \mathrm{~cm})$.

The forearm was placed on the same rig utilised for the surrogate testing. A piece of adhesive foam (Swanfoam, $\mathrm{L}$ $38 \mathrm{~cm} \times \mathrm{W} 22.5 \mathrm{~cm} \times \mathrm{H} 0.3 \mathrm{~cm}$ ) was placed between the forearm and rig to improve comfort and reduce movement of the forearm during testing. Similar to the surrogate testing, wrist angular position was recorded at $60 \mathrm{fps}$ using the 
digital camcorder positioned with the image plane parallel to the plane of motion of the wrist joint from a distance of $150 \mathrm{~cm}$. Three self-adhesive coloured markers $(6 \mathrm{~mm}$ diameter) were attached (Fig. 3) to enable wrist angles of flexion-extension to be obtained from the video footage, on bone landmarks; lateral placement over the Triquetrum, tip of 5th metacarpal, and lateral epicondyle [36]. For ulnarradial deviation three similar coloured markers were also used, with the forearm positioned in mid-pronation, on bone landmarks; dorsal Capitate bone, 3rd metacarpal bone, dorsal midline of the forearm [36]. For all motions, participants were instructed to move only in the requested plane of movement without any deviation.

The participants started with the left forearm placed in a full pronated position. The hand was positioned neutral

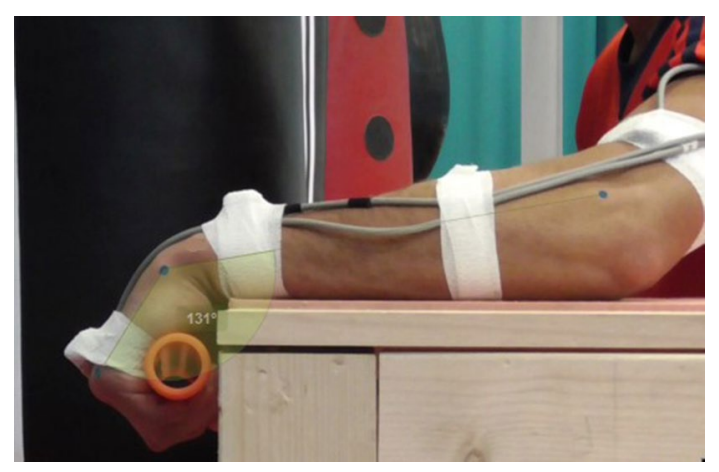

Fig. 3 Kinovea software analysis showing an example of quasistatic testing for flexion. Three self-adhesive markers (blue) can be observed; at the hand, wrist and elbow (colour figure online) with a closed fist holding onto a plastic cylindrical handle (L $12 \mathrm{~cm} \times \mathrm{D} 4 \mathrm{~cm}$ ), to mimic the functional position of a boxer's hand when held in a glove. The participant was asked to fully flex at the wrist, hold the position for $3 \mathrm{~s}$, fully extend at the wrist and again hold still for $3 \mathrm{~s}$. For ulnar and radial deviation, the same procedure was used with the forearm positioned in mid-pronation. Out of plane movement was limited by instructions provided to participants. All motions were performed three times. A frame was collected at the static angle that the wrist was held at for each motion, extracted from the video recording, and analysed using the Kinovea software program (Fig. 3). The wrist angles obtained were used for further statistical analysis. Similar to the surrogate testing, to measure test-retest reliability for flexion-extension the system was recalibrated, and the data collected again. This procedure was equally performed for measuring ulnar-radial deviations.

\subsubsection{Punch testing}

The electromagnetic tracking system receivers were fixed to the left upper limb following the same procedure for the quasi-static testing (Figs. 2, 4a). A traditional cotton boxing wrap ( $\mathrm{L} 450 \mathrm{~cm} \times \mathrm{W} 5 \mathrm{~cm}$ ) was used to bandage the left hand of each participant using a standard technique (Fig. 4b). This bandage was not used in the quasi-static testing due to the requirement for self-adhesive markers and subsequent video analysis. To minimise the risk of knuckle injuries during testing, a piece of foam (Hapla Swanfoam, Cuxson Gerrard \& Co. Ltd., L $10 \mathrm{~cm} \times \mathrm{W} 4 \mathrm{~cm} \times \mathrm{H} 0.6 \mathrm{~cm}$ ) was placed directly over the anterior aspect of metacarpals, as typically
Fig. 4 Electromagnetic tracking system receiver placement; a on the hand and forearm for both the quasi-static and punch testing, $\mathbf{b}$ with the addition of the standard bandage technique covering the receivers, $\mathbf{c}$ with the addition of boxing gloves covering the standard bandage technique and receivers, and d during punch testing in the boxing gym
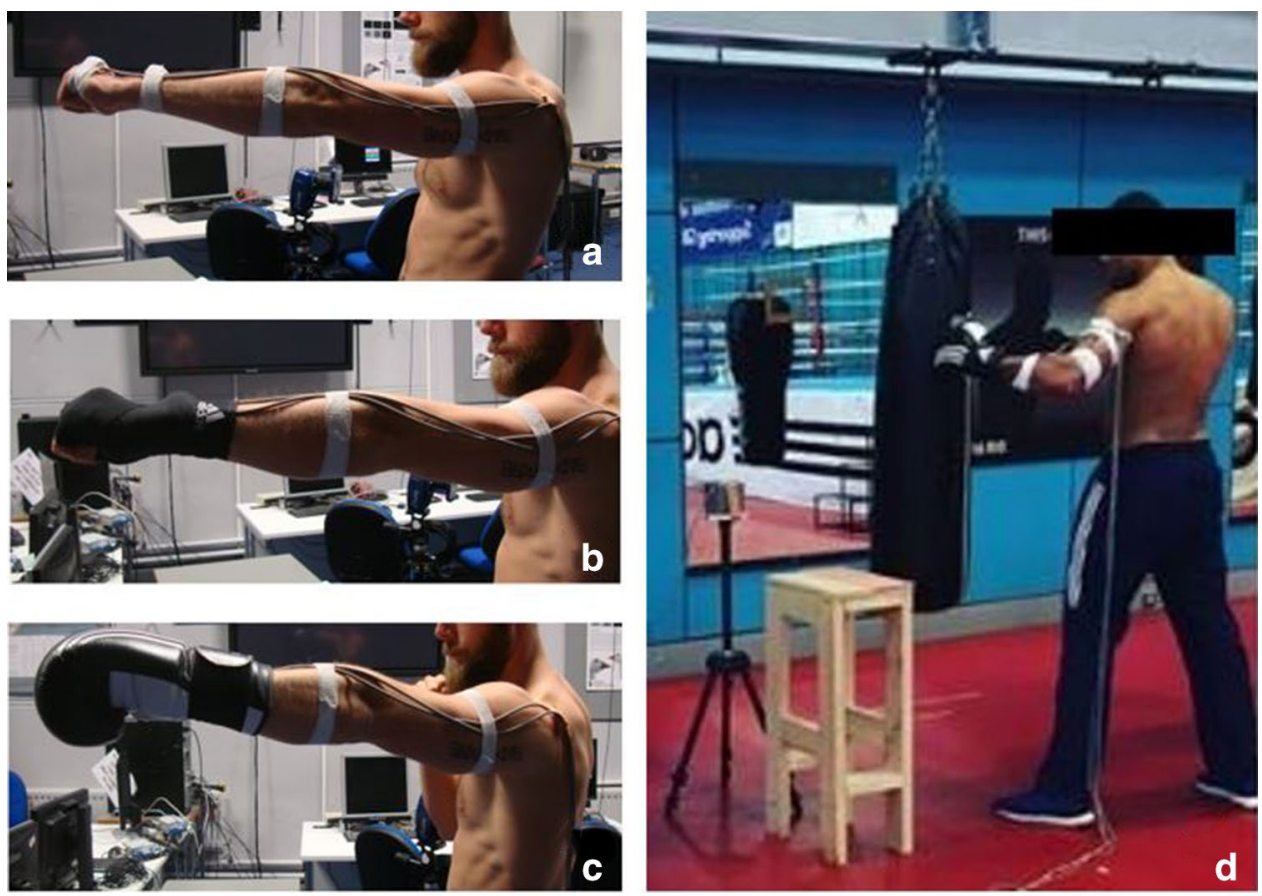
used by boxers during training. A boxing glove (14 oz Adidas), of the correct size, was worn by each participant covering the hand and forearm receivers (Fig. 4c).

The participants were asked to face a boxing bag (Adidas heavy bag), adopting their natural orthodox stance (Fig. 4d). Each participant was then asked to throw two types of commonly used shots in boxing with their lead hand; jab (straight shot) and hook (bent arm shot). Jab shots were performed six times, allowing a rest period of approximately three seconds between shots. The 2 nd to 5 th shots were used for statistical analysis to assess reliability. The 1 st and 6th shot were not analysed, to limit potential errors from the shots thrown by boxers at the beginning and end of testing. The same procedure was repeated for hook shots.

\subsection{Data analysis and angle definition}

The electromagnetic tracking system data from all testing procedures was processed using Visual 3D v3.79 (C-Motion, Germantown, MD, USA). Following a comparable protocol to Schmitz et al. [37], marker trajectories were filtered using the a lowpass fourth order zero-lag Butterworth filter in visual 3D, using $10 \mathrm{~Hz}$ as the cut-off frequency defined through visual inspection of the fit during pilot testing distinguishing between noise (e.g. glove vibration/movement) and true measurements during the impact phase. The body-fixed reference frames were then constructed using the marker positions. The filtered trajectories of the digital markers were subsequently used to compute orientation of the distal segment relative to the proximal segment using Cardan angles [38]. Positive and negative rotations around the $x$-axis were defined as flexion and extension, respectively (Fig. 5). Positive and negative rotations around the $y$-axis were defined as radial deviation and ulnar deviation, respectively (Fig. 5). For the surrogate and quasi-static testing an event marker was created corresponding to the maximum and minimum points of all four wrist motions; flexion, extension, ulnar deviation and radial deviation (Fig. 5a).

For the punch testing, it was important to define the wrist angle (Fig. 5b) being calculated (i.e. the wrist angle at impact with the bag). The wrist angle was identified using three combined methods; (1) visual observation of the virtual upper limb (tested at $240 \mathrm{~Hz}$ ) to identify the point of hand impact observed at terminal elbow extension combined with terminal shoulder flexion, (2) movement at the $x$-axis and $y$-axis aligned together with displacement observed to occur simultaneously at the perceived point of hand impact, and (3) movement at the $x$-axis aligned with the acceleration of the wrist with the maximum acceleration observed to occur simultaneously with maximum $x$-axis displacement. While the markers at the arm for the participants were not directly required for wrist measurements, they were utilised to assist visual observation of the virtual upper limb when identifying the wrist angle at impact. For the punch testing,

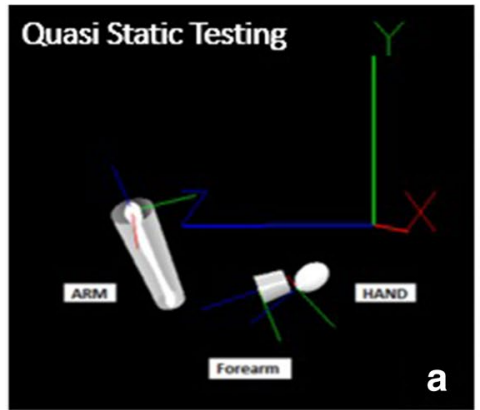

a
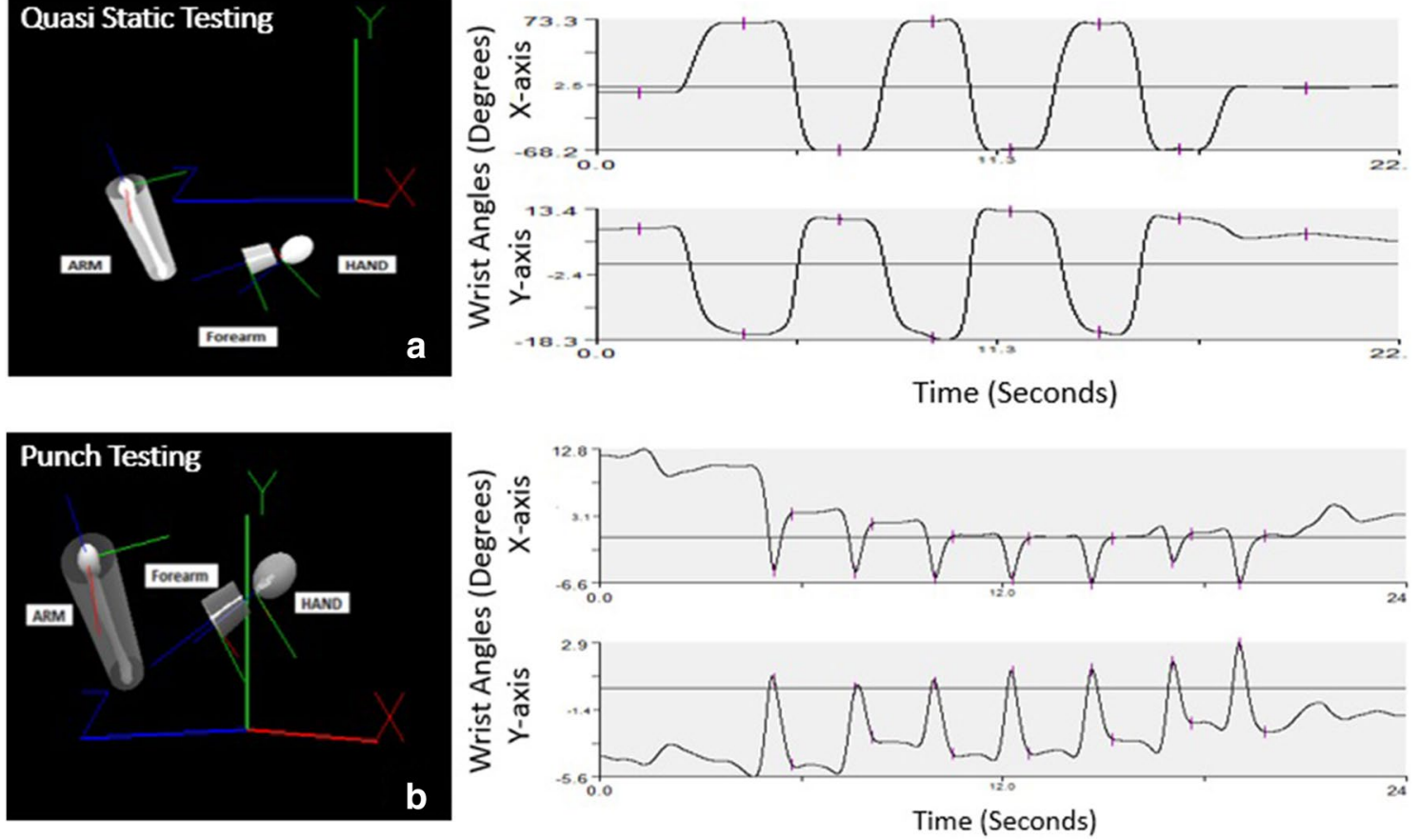

Fig. 5 Visual 3D software: on the left, computer generated model showing individual anatomical segments; arm, forearm, hand. On the right, flexion-extension ( $x$-axis) and ulnar-radial deviations ( $y$-axis) wrist angles with event markers (red) created for; a quasi-static testing and $\mathbf{b}$ punch testing; jab shot (colour figure online) 
an event marker was created corresponding to the maximum and minimum points of all four wrist motions occurring at impact with the bag; flexion, extension, ulnar deviation and radial deviation (Fig. 5b).

For the accuracy of the electromagnetic tracking system in the surrogate and quasi-static testing a paired $t$ test was used. To further assess the agreement of the electromagnetic tracking system with the video-based system in the quasi static testing, Bland-Altman analysis was performed for each of the four motions tested; flexion, extension, ulnar deviation and radial deviation. Intra-participant reliability of each measure for all tests performed (i.e. surrogate, quasistatic, and punch) was examined using the intraclass correlation coefficient (ICC). The following benchmarks for ICC for reliability were used: Poor (0.00-0.20), Fair (0.21-0.40), Moderate (0.41-0.60), Substantial (0.61-0.80), and Good (0.81-1.00) agreement [39].

\section{Results}

Wrist angles calculated from the electromagnetic tracking system deviated from the video-based system by $<0.2^{\circ}$ for flexion-extension in the surrogate testing, and $<6^{\circ}$ for flexion-extension and ulnar-radial deviations in the quasi static testing (Table 1). The electromagnetic tracking system showed better agreement with the video-based system at estimating ulnar-radial deviations $\left(<4^{\circ}\right.$ mean difference) than flexion-extension deviations $\left(<6^{\circ}\right.$ mean difference) in the quasi static testing (Table 1). The limits of agreement met the assumptions of Bland-Altman analysis (Fig. 6), ranging from $0.4^{\circ}$ to $11.1^{\circ}$ which means the model can be used when the acceptable difference from the video-based system is within this range. In all motions, the errors did not appear to be influenced by the mean angle between the electromagnetic tracking system and the video-based system.

The electromagnetic tracking system demonstrated good reliability (ICCs > 0.9) [39] for both the surrogate and quasistatic data (Table 2). Jab and hook shots for wrist motions occurring during the punch testing in flexion-extension yielded good reliability (ICCs $>0.8$ ) [39], whilst a substantial reliability (ICCs $>0.6$ ) [39] for both types of shots for wrist motions was recorded in ulnar-radial deviations (Table 3).

\section{Discussion}

When testing a rigid surrogate wrist held at different angles, which eliminates potential errors of anatomy and skin movement, joint angles measured with the electromagnetic tracking system agreed with the video-based system within $<0.2^{\circ}$. For the quasi-static measurement of boxer's wrists, the electromagnetic tracking system agreed with the videobased system within $2^{\circ}-6^{\circ}$ for all four movements tested, with the largest difference of $5.7^{\circ}$ similar to reported maximum differences of $5^{\circ}$ observed in other clinical studies [40, 41]. Agreement between the electromagnetic tracking system and video-based system in the quasi-static testing was further confirmed with Bland-Altman analysis [42, 43]. The largest difference between the two systems was observed to occur during flexion testing. The electromagnetic receivers were placed on the dorsum of the hand, whilst the selfadhesive markers for video capturing were placed on the medial aspect, therefore providing an explanation for the larger difference obtained during flexion compared with the other motions. Considering that the hand was maintained in a fist position, it was expected that the overlying structures (skin and underlying fascia) on the dorsum of the fingers, hand and wrist would be more stretched when compared to the volar, medial and lateral aspects. The bandage, utilised for the punch testing, would have potentially reduced movement of the electromagnetic receivers on the underlying skin due to direct pressure onto these receivers. The use of the bandage during the quasi-static method was, however, not viable due to the requirement of the self-adhesive markers to be placed on the skin and captured with the video camera. Securing the receivers during the punch testing was important and, therefore, considered when bandaging the handwrist complex.

Another consideration is that substantial inter-carpal joint movements occur in the region of the wrist [44-46]. Several authors have stated that the centre of rotation for the wrist occurs at the Capitate bone [47-49]. In a study using
Table 1 Accuracy of the electromagnetic tracking system for the surrogate and quasistatic testing (units in degrees)

\begin{tabular}{lllllll}
\hline & Motion & Correlation & Sig & Mean difference & Std. deviation & Std. error mean \\
\hline Surrogate & FLEX-EXT & 1 & $\mathbf{> 0 . 0 0 1}$ & 0.12 & 0.08 & 0.03 \\
Quasi-static & FLEX & 0.973 & $\mathbf{> 0 . 0 0 1}$ & 5.73 & 2.73 & 0.51 \\
& EXT & 0.998 & $\mathbf{> 0 . 0 0 1}$ & 2.46 & 0.83 & 0.15 \\
& UD & 0.986 & $\mathbf{> 0 . 0 0 1}$ & 3.52 & 1.62 & 0.3 \\
& RD & 0.989 & $\mathbf{> 0 . 0 0 1}$ & 2.15 & 0.93 & 0.17 \\
\hline
\end{tabular}

FLEX flexion, EXT extension, $U D$ ulnar deviation, $R D$ radial deviation Significant $p$ values are emboldened 

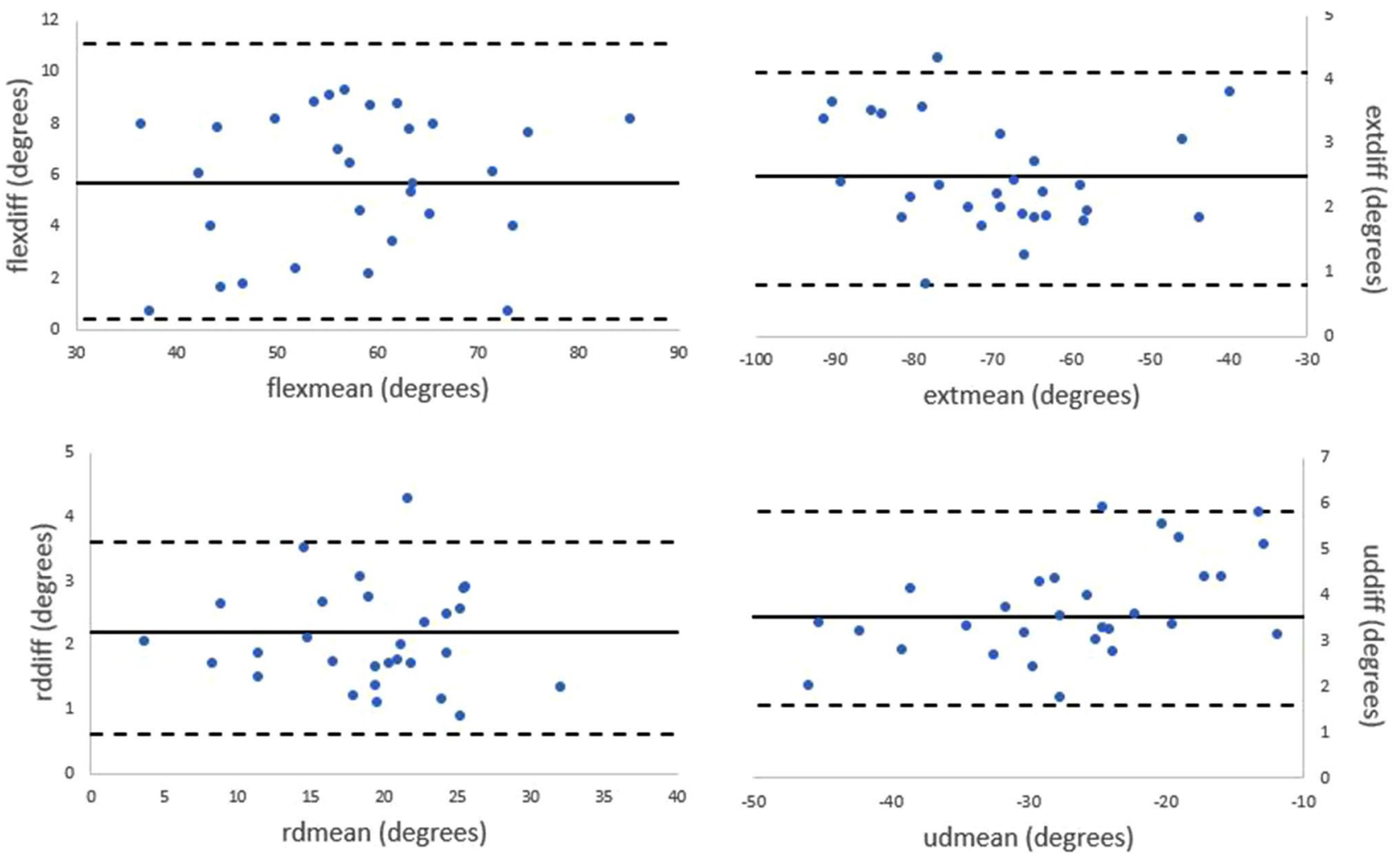

Fig. 6 Bland-Altman analysis for the quasi-static testing (units in degrees); flexion (flex), extension (ext), ulnar deviation (ud), radial deviation (rd)

Table 2 Reliability and 95\% limit of agreement of the motion capture systems (units in degrees)

\begin{tabular}{|c|c|c|c|c|c|c|}
\hline & Motion & $1 \mathrm{st}$ & 2nd & $3 \mathrm{rd}$ & ICC & $95 \%$ CI (\%) \\
\hline Surrogate (video system) & FLEX-EXT & & & & 1 & $0.999-1.000$ \\
\hline Surrogate (electromagnetic tracking system) & FLEX-EXT & & & & 1 & $0.999-1.000$ \\
\hline \multirow[t]{4}{*}{ Quasi-static (video system) } & FLEX & $58.3 \pm 11.9$ & $59.1 \pm 12.1$ & $59.2 \pm 12.0$ & 0.976 & $0.957-0.988$ \\
\hline & EXT & $69.5 \pm 13.0$ & $69.1 \pm 13.7$ & $69.3 \pm 13.2$ & 0.987 & $0.975-0.973$ \\
\hline & UD & $26.1 \pm 9.4$ & $26.6 \pm 9.6$ & $26.5 \pm 9.5$ & 0.967 & $0.941-0.983$ \\
\hline & $\mathrm{RD}$ & $19.0 \pm 6.3$ & $19.8 \pm 6.1$ & $19.9 \pm 6.5$ & 0.970 & $0.946-0.985$ \\
\hline \multirow[t]{4}{*}{ Quasi-static intrasession (electromagnetic tracking system) } & FLEX & $52.6 \pm 11.6$ & $53.3 \pm 11.9$ & $53.5 \pm 12.0$ & 0.961 & $0.930-0.980$ \\
\hline & EXT & $71.7 \pm 13.3$ & $71.7 \pm 13.8$ & $71.8 \pm 13.3$ & 0.990 & $0.982-0.995$ \\
\hline & UD & $29.7 \pm 8.9$ & $30.0 \pm 9.2$ & $30.1 \pm 8.7$ & 0.970 & $0.944-0.985$ \\
\hline & $\mathrm{RD}$ & $17.0 \pm 6.1$ & $17.7 \pm 6.2$ & $17.6 \pm 6.6$ & 0.973 & $0.951-0.987$ \\
\hline \multirow[t]{4}{*}{ Quasi-static intersession (electromagnetic tracking system) } & FLEX & & & & 0.992 & $0.983-0.996$ \\
\hline & EXT & & & & 0.996 & $0.991-0.998$ \\
\hline & UD & & & & 0.941 & $0.879-0.972$ \\
\hline & $\mathrm{RD}$ & & & & 0.974 & $0.945-0.988$ \\
\hline
\end{tabular}

FLEX flexion, EXT extension, $U D$ ulnar deviation, $R D$ radial deviation

high speed video data acquisition for 3D range of movement analysis of a cadaveric wrist, it was observed that during wrist flexion-extension, the instantaneous screw axis was found to qualitatively pass through the head of the Capitate, however, it was not limited or fixed to the Capitate [50]. Patterson and colleagues [50] maintain that centre of rotation calculations assume planar motion and do not account for slippage between the carpal bones during normal carpal 
Table 3 Reliability for wrist motion (units in degrees) at the impact phase for the 2nd-5th Jab and Hook shots

\begin{tabular}{llllllll}
\hline Shot & Motion & 2nd & 3rd & 4th & 5th & ICC & 95\% CI (\%) \\
\hline JAB & FLEX-EXT & $7.5 \pm 4.3$ & $7.9 \pm 4.6$ & $7.8 \pm 5.2$ & $7.2 \pm 4.7$ & 0.850 & $0.757-0.918$ \\
JAB & UD-RD & $3.0 \pm 1.8$ & $2.8 \pm 1.8$ & $2.9 \pm 2.1$ & $2.4 \pm 2.1$ & 0.679 & $0.522-0.813$ \\
HOOK & FLEX-EXT & $4.8 \pm 2.7$ & $4.3 \pm 2.7$ & $5.0 \pm 3.7$ & $4.8 \pm 3.2$ & 0.805 & $0.692-0.892$ \\
HOOK & UD-RD & $2.1 \pm 1.8$ & $1.9 \pm 2.0$ & $1.7 \pm 1.5$ & $1.8 \pm 1.4$ & 0.700 & $0.549-0.827$ \\
\hline
\end{tabular}

FLEX flexion, EXT extension, $U D$ ulnar deviation, $R D$ radial deviation motion. To potentially provide a better comparison, a fourpoint vide-based system could be considered based on the understanding that the wrist is more complicated and modelling it as a fixed hinge joint might not be anatomically correct. In the current study, the electromagnetic tracking system agreed with a three-point video-based system, with the axis of rotation considered at the Capitate. While care was taken in positioning the camera, the video-based system could present a potential source of error due to cross talk between flexion-extension and ulnar-radial deviations resulting in some out of plane motion. Future work could consider using three-dimensional analysis techniques, such as stereo calibrated cameras or a commercial marker-based motion capture system.

During wrist motion, the radio-lunate joint contributes more motion in flexion than the Capito-Lunate joint, with the opposite occurring in extension [50]. These underlying biomechanical differences, combined with potential skin movement, can contribute to variations with repeated movements. In the current study, intra-rater reliability of the video-based system was evaluated by measurement of the wrist joint range of motion. The ICC for all four motions was in a range of $0.967-0.976$ indicating good reliability (ICCs > 0.9) [39], comparable to another study using a similar methodology [35].

Testing the accuracy of the electromagnetic tracking system during punching was not possible, the information from the surrogate and quasi-static testing was therefore considered. For repeatability of the electromagnetic tracking system during punching, reliability of two commonly used shots in boxing (jab and hook) was performed. For both types of shots, flexion-extension yielding better reliability (ICC range; 0.805-0.850) than ulnar-radial deviations (ICC range; $0.679-0.700)$. The difference in reliability between flexion-extension and ulnar-radial deviations potentially attributed to errors with the video-based system. Compared to gait analysis in the lower limb, motion analysis of the upper limb carries several disadvantages. Mainly that there is no single relevant functional activity for upper limb, and that functional activities in this region show a larger variation of execution in the normal population as opposed to gait patterns [11]. Boxing is not considered an activity of daily living and, therefore, not an area that is widely understood and researched. In boxing, the objective is to restrict movement at the wrist to improve transference of forces occurring from the lower limb and trunk towards the upper limb, and into the opponent. This movement restriction at the wrist is also important to decrease injuries occurring to the boxer, evident from the common practice of wrapping hands for both training and competition. Conversely, in activities of daily living and other sports, it is often important for motion to occur in the wrist joint. This current study was, therefore, important in identifying a technology that can measure what level of wrist motion occurs during impact in boxing, whilst still utilising the wrapping material and gloves required.

\section{Conclusion}

The advent of improved technology makes the use of 3D motion capture systems a possibility in quantifying joint movements during sporting activities. A protocol using an electromagnetic tracking system was developed with the results indicating it as an accurate and reliable tool for assessing wrist kinematics in boxing, specifically during repeated punching situations. These results potentially support the use of electromagnetic motion capture devices for a variety of sporting and non-sporting clinical applications. Further studies using such technology are therefore warranted, especially in the upper limb.

Acknowledgements This work was funded in part by the Centre for Sports Engineering Research at Sheffield Hallam University (SHU) and part by the English Institute of Sport (EIS). The authors would like to thank Caroline Adams (PhD student at SHU) for her assistance with the construction of the polyamide surrogate.

\section{Compliance with ethical standards}

Conflict of interest The authors declare they have no conflict of interest. Tom Allen is an editor of the journal and he was not involved in the review and decision-making process for this paper.

Open Access This article is distributed under the terms of the Creative Commons Attribution 4.0 International License (http://creativeco mmons.org/licenses/by/4.0/), which permits unrestricted use, distribution, and reproduction in any medium, provided you give appropriate credit to the original author(s) and the source, provide a link to the Creative Commons license, and indicate if changes were made. 


\section{References}

1. Loosemore M, Lightfoot J, Gatt I, Hayton M, Beardsley C (2017) Hand and wrist injuries in elite boxing: a longitudinal prospective study (2005-2012) of the Great Britain Olympic Boxing Squad. Hand 12:181-187. https://doi.org/10.1177/1558944716642756

2. Loosemore M, Lightfoot J, Palmer-Green D, Gatt I, Bilzon J, Beardsley C (2015) Boxing injury epidemiology in the Great Britain team: a 5-year surveillance study of medically diagnosed injury incidence and outcome. Br J Sports Med 49:1100-1107. https://doi.org/10.1136/bjsports-2015-094755

3. Hall JG, Allanson JE, Gripp KW, Slavotinek AM (2007) Handbook of physical measurements, 2nd edn. Oxford University Press, Oxford

4. Piorkowski BA, Lees A, Barton GJ (2011) Single maximal versus combination punch kinematics. Sports Biomech 10:1-11. https:// doi.org/10.1080/14763141.2010.547590

5. Whiting WC, Gregor RJ, Finerman GA (1988) Kinematic analysis of human upper extremity movements in boxing. Am J Sports Med 16:130-136. https://doi.org/10.1177/036354658801600207

6. Wu G, van der Helm FCT, Veeger HEJ, Makhsous M, Van Roy P, Anglin C, Nagels J, Karduna AR, McQuade K, Wang X, Werner FW, Buchholz B (2005) ISB recommendation on definitions of joint coordinate systems of various joints for the reporting of human joint motion-part II: shoulder, elbow, wrist and hand. J Biomech 38:981-992. https://doi.org/10.1016/j.jbiom ech.2004.05.042

7. Coburn JC, Upal MA, Crisco JJ (2007) Coordinate systems for the carpal bones of the wrist. J Biomech 40:203-209. https://doi. org/10.1016/j.jbiomech.2005.11.015

8. Rab G, Petuskey K, Bagley A (2002) A method for determination of upper extremity kinematics. Gait Posture 15:113-119. https:// doi.org/10.1016/S0966-6362(01)00155-2

9. Roux E, Bouilland S, Godillon-Maquinghen A-P, Bouttens d (2002) Evaluation of the global optimisation method within the upper limb kinematics analysis. J Biomech 35:1279-1283. https ://doi.org/10.1016/S0966-6362(01)00155-2

10. Schmidt R, Disselhorst-Klug C, Silny J, Rau G (1999) A markerbased measurement procedure for unconstrained wrist and elbow motions. J Biomech 32:615-621. https://doi.org/10.1016/S0021 -9290(99)00036-6

11. van Andel CJ, Wolterbeek N, Doorenbosch CAM, Veeger DHEJ, Harlaar J (2008) Complete 3D kinematics of upper extremity functional tasks. Gait Posture 27:120-127. https://doi.org/10.1016/j. gaitpost.2007.03.002

12. Murgia A, Kyberd PJ, Chappell PH, Light CM (2004) Marker placement to describe the wrist movements during activities of daily living in cyclical tasks. Clin Biomech (Bristol, Avon) 19:248-254. https://doi.org/10.1016/j.clinbiomech.2003.11.012

13. Su F-C, Chou YL, Yang CS, Lin GT, An KN (2005) Movement of finger joints induced by synergistic wrist motion. Clin Biomech (Bristol, Avon) 20:491-497. https://doi.org/10.1016/j.clinbiomec h.2005.01.002

14. Asundi K, Johnson PW, Dennerlein JT (2011) Does elevating and tilting the input device support surface affect typing force and postural exposures of the wrist? Work 39:187-193. https://doi. org/10.3233/WOR-2011-1165

15. Fagarasanu M, Kumar S, Narayan Y (2004) Measurement of angular wrist neutral zone and forearm muscle activity. Clin Biomech (Bristol, Avon) 19:671-677. https://doi.org/10.1016/j.clinbiomec h.2004.05.004

16. Michel FI, Schmitt KU, Greenwald RM, Russell K, Simpson FI, Schulz D, Langrin M (2013) White Paper: functionality and efficacy of wrist protectors in snowboarding-towards a harmonized international standard. Sport Eng 16:197-210. https://doi. org/10.1007/s12283-013-0113-3

17. Greenwald RM, Simpson FH, Michel FI (2013) Wrist biomechanics during snowboard falls. Proc Inst Mech Eng Part P J Sport Eng Technol 227:244-254. https://doi.org/10.1177/175433711348270 6

18. Aizawa J, Masuda T, Hyodo K, Jinno T, Yagishita K, Naamaru K, Koyama T, Morita S (2013) Ranges of active joint motion for the shoulder, elbow, and wrist in healthy adults. Disabil Rehabil 35:1342-1349. https://doi.org/10.3109/09638288.2012.731133

19. Heneghan NR, Hall A, Hollands M, Balanos GM (2009) Stability and intra-tester reliability of an in vivo measurement of thoracic axial rotation using an innovative methodology. Man Ther 14:452-455. https://doi.org/10.1016/j.math.2008.10.004

20. Delorme $S$, Tavoularis S, Lamontagne $M(2005)$ Kinematics of the ankle joint complex in snowboarding. J Appl Biomech 21(4):394 403. https://doi.org/10.1123/jab.21.4.394

21. Veeger HEJ, Yu B, An KN, Rozendal RH (1997) Parameters for modelling the upper extremity. J Biomech 30:647-652. https:// doi.org/10.1016/S0021-9290(97)00011-0

22. Hansson GA, Balogh I, Ohlsson K, Rylander L, Skerfving S (1996) Goniometer measurement and computer analysis of wrist angles and movements applied to occupational repetitive work. J Electromyogr Kinesiol 6:23-35. https://doi.org/10.1016/10506411(95)00017-8

23. Buchholz B, Wellman H (1997) Practical operation of a biaxial goniometer at the wrist joint. Hum Factors 39:119-129. https:// doi.org/10.1518/001872097778940696

24. Johnson PW, Jonsson P, Hagberg M (2002) Comparison of measurement accuracy between two wrist goniometer systems during pronation and supination. J Electromyogr Kinesiol 12:413-420. https://doi.org/10.1016/S1050-6411(02)00031-7

25. Smith MS, Dyson RJ, Hale T, Janaway L (2000) Development of a boxing dynamometer and its punch force discrimination efficacy. $\mathrm{J}$ Sports Sci 18:445-450. https://doi.org/10.1080/026404100500743 77

26. Roetenberg D, Baten CTM, Veltink PH (2007) Estimating body segment orientation by applying inertial and magnetic sensing near ferromagnetic materials. IEEE Trans Neural Syst Rehabil 15:469-471. https://doi.org/10.1109/TNSRE.2007.903946

27. Worsey MT, Espinosa HG, Shepherd JB, Thiel DV (2019) Inertial sensors for performance analysis in combat sport: a systematic review. Sports 7:1-19. https://doi.org/10.3390/sports7010028

28. Shepherd JB, Thiel DV, Espinosa HG (2017) Evaluating the use of inertial-magnetic sensors to assess fatigue in boxing during intensive training. IEEE Sens Lett 1:1-4. https://doi.org/10.1109/ LSENS.2017.2689919

29. Kimm D, Thiel DV (2015) Hand speed measurement in boxing. Procedia Eng 112:502-506. https://doi.org/10.1016/j.proen g.2015.07.232

30. Lopez-Nava IH, Angelica MM (2016) Wearable inertial sensors for human motion analysis: a review. IEEE Sens J 99:1-4. https:// doi.org/10.1109/JSEN.2016.2609392

31. Huang B, Giggins O, Kechadi T, Caulfield B (2016) The limb movement analysis of rehabilitation exercises using wearable inertial sensors. Proc Annu Int Conf IEEE Eng Med Biol Soc (EMBC) 2016:4686-4689. https://doi.org/10.1109/EMBC.2016.7591773

32. Chen P, Li J, Luo M, Zhu N (2015) Real-time human motion capture driven by a wireless sensor network. Int J Comput 8:1-14. https://doi.org/10.1155/2015/695874

33. Metcalf CD, Notley SV, Chappel PH, Burridge JH, Yule VT (2008) Validation and application of a computational model for wrist and hand movements using surface markers. IEEE Trans Biomed Eng 55:1199-1210. https://doi.org/10.1109/ TBME.2007.908087 
34. European Committee for Standardization (2003) Protective clothing-wrist, palm, knee and elbow protectors for users of roller sports equipment-requirements and test methods; EN 14120:2003. European Committee for Standardization, Brussels. https://www.document-center.com/standards/show/BS-EN-14120

35. Gajdosik RL, Bohannon RW (1987) Clinical measurement of range of motion: review of goniometry emphasizing reliability and validity. Phys Ther 67:1867-1872. https://doi.org/10.1093/ $\mathrm{ptj} / 67.12 .1867$

36. El-Raheem RMA, Kamel RM, Ali MF (2015) Reliability of using Kinoveo program in measuring dominant wrist joint range of motion. Trends Appl Sci Res 10(4):224-230. https://doi. org/10.3923/tasr.2015.224.230

37. Schmitz A, Ye M, Shapiro R, Yang R, Noehren B (2014) Accuracy and repeatability of joint angles measured using a single camera markerless motion capture system. J Biomech 47:587-591. https ://doi.org/10.1016/j.jbiomech.2013.11.031

38. Grood ES, Suntay WJ (1983) A joint coordinate system for the clinical description of three-dimensional motions: application to the knee. J Biomech Eng 105:136. https://doi.org/10.1115/1.31383 97

39. Landis JR, Koch GG (1977) The measurement of observer agreement for categorical data. Biometrics 33:159. https://doi. org/10.2307/2529310

40. Butler RJ, Barrios JA, Royer T, Davis IS (2011) Frontal-plane gait mechanics in people with medial knee osteoarthritis are different from those in people with lateral knee osteoarthritis. Phys Ther 91:1235-1243. https://doi.org/10.2522/ptj.20100324

41. Noehren B, Pohl MB, Sanchez Z, Cunnigham T, Lattermann C (2012) Proximal and distal kinematics in female runners with patellofemoral pain. Clin Biomech 27:366-371. https://doi. org/10.1016/j.clinbiomech.2011.10.005

42. Field A (2014) Discovering statistics using IBM SPSS statistics, 4th edn. SAGE Publications, London
43. Bland JM, Altman DG (2003) Applying the right statistics: analyses of measurement studies. Ultrasound Obstet Gynecol 22:85-93. https://doi.org/10.1002/uog.122

44. Sun JS, Shih TT, Ko CM, Chang CH, Hang YS, Hou SM (2000) In vivo kinematic study of normal wrist motion: an ultrafast computed tomographic study. Clin Biomech (Bristol, Avon) 15:212216. https://doi.org/10.1016/S0268-0033(99)00064-9

45. Gellman H, Kaufman D, Linihan M (1988) An in vitro analysis of wrist motion: the effect of limited intercarpal arthrodesis and the contributions of the radiocarpal and midcarpal joints. J Hand Surg Am 13:378-383. https://doi.org/10.1016/S0363-5023(88)80013-3

46. de Lange A, Kauer JMG, Huiskes R (1985) Kinematic behavior of the human wrist joint: a roentgen-stereophotogrammetric analysis. J Orthop Res 3:56-64. https://doi.org/10.1002/jor.1100030107

47. Jackson WT, Hefzy MS, Guo HQ (1994) Determination of wrist kinematics using a magnetic tracking device. Med Eng Phys 16:123-133. https://doi.org/10.1016/1350-4533(94)90025-6

48. Brumbaugh RB, Crowninshield RD, Blair WF, Andrews JG (1982) An in vivo study of normal wrist kinematics. J Biomech Eng 104:176-181. https://doi.org/10.1115/1.3138345

49. Andrews JG, Youm Y (1979) A biomechanical investigation of wrist kinematics. J Biomech 12(1):83-93. https://doi. org/10.1016/0021-9290(79)90012-5

50. Patterson RM, Nicodemus CL, Viegas SF, Elber KW, Rosenblatt J (1998) High-speed, three-dimensional kinematic analysis of the normal wrist. J Hand Surg Am 23:446-453. https://doi. org/10.1016/S0363-5023(05)80462-9

Publisher's Note Springer Nature remains neutral with regard to jurisdictional claims in published maps and institutional affiliations. 